\title{
Gestão dos Recursos Hídricos em Bacias Hidrográficas: Monitoramento Hidrológico, uma Integração Participativa
}

\author{
Ancelmo Arantes Valente ${ }^{1}$ \\ Edna Regina Gobbo César ${ }^{1}$ \\ Danilo Augusto Faria ${ }^{3}$ \\ Marli Aparecida Reis Maciel Leite ${ }^{2}$ \\ Paulo Augusto Romera e Silva ${ }^{1}$ \\ ${ }^{1}$ Centro Tecnológico de Hidráulica e Recursos Hídricos - CTH/DAEE \\ Av. Prof. Lúcio Martins Rodrigues, 120 - Butantã - São Paulo \\ São Paulo - Brasil - CEP 05508-900 \\ \{ancelmo,romera\}@cth.usp.br \\ edna.cesar@daee.sp.gov.br \\ ${ }^{2}$ Comitê da bacia Hidrográfica do Paraíba do Sul - CBH-PS \\ Largo Santa Luzia, 25 - Taubaté/São Paulo - Brasil - CEP 12010-510 \\ cbh-ps@uol.com.br \\ ${ }^{3}$ Diretoria da Bacia do Paraíba e Litoral Norte - BPB/DAEE \\ Largo Santa Luzia, 25 - Taubaté/São Paulo - Brasil - CEP 12010-510 \\ danilo.faria@daee.sp.gov.br
}

\begin{abstract}
S This article has origin in research entitled, "CONFLICT MINIMIZATION IN THE USE OF WATER RESOURCES BY HYDRAULIC ENGINEERING MANAGEMENT: A study of the Hydrographic Basin of the Pirapitingüi Brook - Valley of the Paraíba" by Ancelmo Arantes Valente. Scope of the research demonstrates the impact of human behavior on hydrographic cycle and volumes of water resulting from the combination of activities and multiple usages. Conclusions are drawn from examples of real conflicts over use of water by scenario simulation, including a proposal for interchange of information between rice farmers and managers, given the character of unique relationship of water in the hydrographic basin and the parameters of Hydraulic Balance. To minimize conflicts, given resource demand versus availability, research was based upon contextualizing several intervening factors in the process of installation, by means of bibliographic search of materials from the most diverse authors and institutions.
\end{abstract}

Palavras-chave: água, gestão, recursos hídricos, Bacia Hidrográfica do Ribeirão Pirapitingüi.

\section{INTRODUÇÃO}

O Ministério do Meio Ambiente - MMA, por meio da Secretaria Nacional de Recursos Hídricos - SRH, quando da divulgação do Plano Nacional de Recursos Hídricos - PNRH (2006), apresentou possíveis cenários nacionais de utilização dos recursos hídricos no Brasil, para 2020 referentes à utilização dos recursos hídricos no Brasil, que dependem do ritmo e da forma de crescimento de seus principais usos: agricultura irrigada, indústria, pecuária, saneamento e geração de energia.

A pesquisa para a construção desses cenários, dentre os fatores que embasaram suas conclusões, enfatiza que "qualquer que seja o cenário, o componente de gestão é decisivo para amenizar problemas, conflitos e melhorar a racionalidade no uso das águas".

Dando continuidade, o PNRH (2006) acrescenta: "além disso, as ações dos usuários poderão ou não ser reguladas e seus impactos sobre os recursos hídricos e entre esses usos e outros poderão ou não ser amenizados, a depender do tipo de gestão que estará vigente no país e do volume de investimentos disponíveis".

Esses cenários foram construídos a partir de uma adaptação da metodologia proposta por Godet (1993), que permite a identificação dos atores-chave, de suas alianças e conflitos, bem 
como do grau de mobilização e de conflitualidade dos objetivos estratégicos para uma mudança desejável, neutralizando ameaças e aproveitando as oportunidades oferecidas por um futuro factível.

Ainda segundo o PNRH (2006), a região hidrográfica do sudeste possuía uma área irrigada, em 2005, de 295 mil ha, e um potencial irrigável de um milhão ha. Para 2020 o mesmo PNRH prevê uma área irrigada da ordem de 500 mil ha. Percentualmente, essa área irrigada será da ordem de $50 \%$ do potencial devendo receber um incremento, no período de 2005/2020, de 69\%. Em termos nacionais, a previsão é que a área irrigada total aumente de 3,6 milhões ha para 5,8 milhões ha, com um incremento de 58\%.

Tal crescimento é motivado pela demanda nacional e mundial de alimentos, pelos preços internacionais e pela maior produtividade alcançada, como resultado de fatores relacionados às novas formas de irrigação e avanços tecnológicos e de manejo mais sensíveis nas regiões com maiores superfícies irrigadas, onde os métodos pressurizados ultrapassam, quanto à área irrigada, os métodos por superfície, com maior controle do uso da água e, portanto, maior eficiência de uso.

A Bacia Hidrográfica do Rio Paraíba do Sul é uma das prioridades do PNRH e pertence a uma região de alta complexidade social, econômica e de grande sentido histórico para o país.

Os municípios que compõem a Unidade de Gerenciamento de Recursos Hídricos UGRHI 2, na qual está inserida a Bacia Hidrográfica do Rio Paraíba do Sul, estão posicionados ao longo do principal eixo econômico do país, formado pelas duas maiores metrópoles do Brasil: São Paulo e Rio de Janeiro. Essa condição geográfica propiciou o surgimento de importantes pólos de desenvolvimento, não só do Estado como também de projeção nacional. São José dos Campos se destaca por possuir um parque industrial diversificado e centros de pesquisa tecnológica, o que lhe confere uma situação privilegiada, não apenas em termos de estrutura produtiva, como também por poder contar com mão-deobra altamente especializada.

Taubaté e Jacareí também têm um conjunto industrial expressivo destacando-se, como principais ramos industriais da UGRHI 2, a aeronáutica, papel e celulose, automobilística, química, mecânica e eletroeletrônica.

A atividade extrativa mineral é recorrente nas áreas de várzeas e as atividades agrícola e pecuária têm mais expressão nos municípios menores, com pouca relevância no contexto do Estado de São Paulo.

Em que pese o fato de a bacia hidrográfica do rio Paraíba do Sul possuir grande volume de informações disponíveis, os conflitos presentes em torno de suas águas ainda são pouco conhecidos, muitas vezes camuflados pelos próprios usuários na intenção de não chamar a atenção dos órgãos gestores.

Entretanto, outros exemplos explícitos de conflito podem ser citados:

- conflitos decorrentes da transposição das vazões do rio Paraíba do Sul para o Sistema Light (bacias dos rios Paraíba do Sul e Piraí);

- conflitos decorrentes da contaminação de mananciais de abastecimento por defensivos agrícolas (ribeirão Guaratinguetá);

- conflitos entre irrigantes devido à ausência de gerenciamento dos recursos hídricos (bacia do rio Piaguí);

- conflito entre irrigantes e outros usuários da água (ribeirão da Serragem); e

- conflitos, causados por excesso de água, comum nos canais, quando o controle das comportas não é realizado de maneira adequada nos períodos de chuva, e causar prejuízos aos proprietários rurais que cercam os corpos hídricos (Campos dos Goytacazes). 


\section{O PROBLEMA}

Ao propósito deste trabalho importou salientar o tópico que se tornou objeto dos estudos, e, de acordo com Cervo e Bervian (2002, p. 73): "o problema levantado deve expressar uma relação entre duas ou mais variáveis. A elaboração clara do problema é fruto da revisão da literatura e da reflexão pessoal". Dessa forma, pôde-se formular claramente a questão central da pesquisa assim delimitada: quais os fatores determinantes para o equilíbrio entre disponibilidade e demanda natural da água, para minimizar os conflitos decorrentes das interferências humanas no ciclo hidrológico?

Para responder a essas indagações foram definidos os cenários em decorrência da demanda versus disponibilidade dos recursos hídricos, com a seguinte descrição:

- cenário espontâneo: onde predominam decisões com base em fatores econômicos sem levar em conta os limites dos recursos naturais;

- cenário induzido: onde devem predominar as decisões com base no princípio da sustentabilidade e no reconhecimento dos limites dos recursos naturais; e

- diferença entre os cenários: uma medida do esforço humano coletivo para passar da situação do cenário espontâneo para a situação do cenário induzido.

De acordo com Braga, Tucci e Porto (2006, p. 146), o planejamento e gestão dos recursos hídricos dependem de informações confiáveis tanto no que diz respeito à demanda como à oferta de água. Essa última só poderá ser adequadamente estimada se existirem redes de monitoramento que gerem dados sobre variáveis que indiquem a quantidade disponível e a respectiva qualidade das águas.

\section{OBJETIVOS}

Esta pesquisa teve por objetivo geral a realização de um estudo descritivo como contribuição para o desenvolvimento institucional das políticas públicas de recursos hídricos em prática no Brasil, por meio da indução e incentivo de ações de gestão integrada na bacia hidrográfica do Ribeirão Pirapitingüi, adotada como estudo de caso.

Buscou-se analisar um exemplo real de conflitos pelo uso da água com a simulação de cenários e a proposta de intercâmbio de informações entre os rizicultores e os gestores, ao levar em conta o caráter de unicidade da água da bacia hidrográfica e os parâmetros do Balanço Hídrico, com vistas à sua minimização, em decorrência da demanda versus disponibilidade dos recursos.

Definiram-se como objetivos específicos, desta pesquisa, diagnosticar e definir os direcionadores para apoiar decisões levando em conta as variáveis do balanço hídrico, que consiste no conhecimento da relação demanda versus disponibilidade, na simulação de cenários e no intercâmbio de informações entre os rizicultores e os gestores, para minimização de conflitos.

O trabalho é intra-regional e está centrado no Vale do Paraíba, Cone Leste Paulista, precisamente no Município de Roseira, na atividade de rizicultura. São levantadas informações referentes aos usuários, instalados na bacia do Ribeirão do Pirapitingüi, para avaliação dos impactos das atividades humanas no ciclo hidrológico e na quantidade das águas decorrentes desse conjunto de atividades, como resultado dos usos múltiplos. Segundo Carrera e Fernandez (2002, p. 105) "o princípio dos usos múltiplos estabelece também uma saudável competição entre os distintos tipos de usuários dos recursos hídricos”.

Neste trabalho discutiram-se os problemas relacionados aos impactos na quantidade da água referente à organização institucional e à legislação brasileira e são apresentadas idéias de longo alcance com influência sobre o universo do planejamento e gerenciamento de seu uso. 


\section{DELIMITAÇÃO DO ESTUDO}

O trabalho tem foco e está delimitado na discussão entre a oferta e a demanda de água superficial e dos instrumentos adequados de gestão, para um desafio que envolve a pequena bacia hidrográfica do Ribeirão Pirapitingüi, que é um afluente do rio Paraíba do Sul, com drenagem em uma área de aproximadamente $116 \mathrm{~km}^{2}$, na qual estão instalados por volta de trinta usuários da água, dentre eles 11 rizicultores.

Define-se como bacia hidrográfica de um rio, em um dado ponto, a superfície de uma área limitada por um contorno, dentro do qual toda a água precipitada, quando não evaporada ou retida, escoa para aquele ponto, já que a rede de drenagem que escoa o fluxo para a seção do rio que a define é função das declividades e do relevo. O que deve sempre ser lembrado é que a bacia hidrográfica é um sistema físico complexo.

\section{RELEVÂNCIA DO ESTUDO}

A orientação que norteia as ações de outorga de direito de uso dos recursos hídricos se baseia na Lei Estadual n ${ }^{\circ}$ 9034, de 27 de dezembro de 1994.

Estabelece que a vazão de referência a ser adotada é a $\mathrm{Q}_{7,10}$ e nas vazões regularizadas por reservatórios, quando a somatória das vazões captadas superar $50 \%$ da vazão de referência, a bacia será decretada crítica e necessitará da intervenção do órgão gestor que lhe dispensará gerenciamento especial.

Constatada esta realidade na bacia hidrográfica do Ribeirão do Pirapitingüi, após a realização de estudos preliminares, o DAEE submeteu ao Comitê da Bacia Hidrográfica do Paraíba do Sul - CBH-PS, a decretação de bacia crítica, em face à disponibilidade hídrica e as demandas existentes.

Assim, levado ao plenário do CBH-PS, em sua $18^{a}$ Reunião Extraordinária, realizada em 24/10/2003, o pleito foi ratificado, quase que por unanimidade.

Pode-se afirmar que a verificação atualizada da disponibilidade hídrica das águas superficiais da bacia hidrográfica do Ribeirão Pirapitingüi, aspecto central do presente estudo, é fundamental para a realização do balanço entre disponibilidade versus demanda, em conjunto com outros estudos em andamento, relativos a esta mesma bacia, que devem constituir-se em um efetivo processo de gestão, de caráter permanente.

Para tanto, considera o contexto passado e atual dos fatores determinantes do desenvolvimento econômico que estabeleceram as formas de apropriação dos recursos naturais como insumos de produção, de modo que os desafios atuais da gestão ambiental e dos recursos hídricos possam:

- gerir situações de conflitos em suas várias formas;

- atuar na sua prevenção em regiões em que há, ou possa haver, desequilíbrios entre a oferta e a demanda de recursos naturais;

- aperfeiçoar os mecanismos institucionais e de legislação, com base na consciência dos novos conhecimentos adquiridos, com novas atitudes e habilidades que possam ser desenvolvidas a partir deles e com a participação da sociedade, para que as soluções encontradas sejam capazes de atender a essas novas exigências.

Para Frederick (1993), a capacidade de gerenciar os inúmeros conflitos resultantes da intensificação das atividades humanas e a degradação dos recursos hídricos é uma preocupação constante de pesquisadores, administradores, gerentes e tomadores de decisão. A partir desse contexto implantaram-se no Brasil e, particularmente, no Estado de São Paulo, um conjunto de macrodiretrizes, sob a forma de políticas públicas, cujos objetivos envolvem a sociedade na percepção, no entendimento dos processos, e em decisões que induzam a uma sustentabilidade no uso dos recursos hídricos, que deram o respaldo necessário ao 
desenvolvimento desta pesquisa.

Para tanto, foram utilizadas informações relativas às áreas irrigadas, sua relação com a disponibilidade hídrica, interação com os rizicultores para a avaliação de cenários e alternativas para regularização da disponibilidade hídrica na bacia, sob a forma de monitoramento hidrológico, com o suporte do órgão gestor dos recursos hídricos do Estado de São Paulo, ficando, neste contexto, evidenciado a relevância do presente estudo, frente aos princípios preconizados pela Política Estadual de Recursos Hídricos.

\section{METODOLOGIA}

O presente trabalho foi realizado na bacia hidrográfica do Ribeirão Pirapitingüi no qual foram aplicados os conceitos anteriormente delineados na revisão da literatura, devendo implementar propostas trabalhadas para a região. A sistemática utilizada foi a da pesquisa descritiva que o qualifica quanto aos fins e quanto aos meios.

Quanto aos fins, a pesquisa foi exploratória e teve como objetivo contribuir para o desenvolvimento e execução da política estadual de recursos hídricos no Estado de São Paulo, com base em definição dada por Cervo e Bervian (2003, p. 69): "os estudos exploratórios não elaboram hipóteses a serem testadas no trabalho, restringindo-se a definir objetivos e buscar mais informações sobre determinado assunto de estudo". Quanto aos meios, ainda segundo Cervo e Bervian (2003, p. 66), "busca conhecer as diversas situações e relações que ocorrem na vida social, política, econômica e demais aspectos do comportamento humano, tanto do indivíduo, tomado isoladamente, como de grupos e comunidades mais complexas". Portanto, envolveu levantamento bibliográfico e estudo de caso para chegar à compreensão de seu objeto como um todo, de acordo com Gil (1996, p. 122).

No entender de Goode e Hatt (1969, p. 422) o método do estudo de caso "não é uma técnica específica. É um meio de organizar dados sociais preservando o caráter unitário do objeto social estudado". De outra forma, Tull (1976, p. 323) afirma que "um estudo de caso refere-se a uma análise intensiva de uma situação particular" e Bonoma (1985, p. 203) coloca que o "estudo de caso é uma descrição de uma situação gerencial".

Ao comparar o método do estudo de caso com outros, Yin (2006, p. 24) afirma que para definir o método a ser usado em uma pesquisa é preciso antes analisar as questões que são colocadas pela investigação. De modo específico, esse método é adequado para responder às questões "quem", "o que", "onde", "como" e "por que" que são questões explicativas e tratam de relações operacionais que ocorrem ao longo do tempo, mais do que freqüências ou incidências.

Ainda de acordo com Yin (2006, p. 26), a preferência pelo estudo de caso deve ser dada nos estudos de eventos contemporâneos, em situações em que os comportamentos relevantes não podem ser manipulados, mas é possível se fazer observações diretas. Portanto, o método do estudo de caso se caracteriza pela "capacidade de lidar com uma ampla variedade de evidências - documentos, artefatos, entrevistas e observações” (Yin, 2006, p. 27).

O método do estudo de caso e outros métodos qualitativos são úteis, segundo Bonoma (1985, p. 207), "quando um fenômeno é amplo e complexo, onde o corpo de conhecimentos existente é insuficiente para permitir a proposição de questões causais e quando um fenômeno não pode ser estudado fora do contexto no qual ele naturalmente ocorre".

Bonoma (1985, p. 206), ao tratar dos objetivos da coleta de dados, também coloca como objetivos do método do estudo de caso, não a sua quantificação ou a sua enumeração, "mas, ao invés disto: descrição; classificação (desenvolvimento de tipologia); desenvolvimento teórico; e o teste limitado da teoria. Em uma palavra, o objetivo é compreensão".

Para a coleta de dados, a metodologia consistiu na busca literária para apreensão do conhecimento científico existente, acerca da dimensão tanto do contexto quanto do fenômeno 
de interesse estudado, seguida de inferência em documentos e observações diretas para argumentação das evidências obtidas. Segundo Cervo e Bervian (2003, p. 28), essa tarefa de coleta de dados, que corresponde a uma fase intermediária da pesquisa, coloca "o observador deliberadamente na posição de observador e de expectador, evitando se envolver ou deixar-se envolver com o objeto da observação".

Por fim, quanto aos meios, a metodologia consistiu em uma pesquisa bibliográfica, documental e observacional, dadas as peculiaridades de constituir-se em estudo sistematizado por meio de técnicas apoiadas nas contribuições teóricas existentes referente à gestão participativa dos recursos hídricos, que disponibilizaram o ferramental analítico necessário para a análise de documentos e registros das observações.

\section{BACIA HIDROGRÁFICA DO RIBEIRÃO PIRAPITINGÜI}

O Ribeirão do Pirapitingüi é afluente pela margem direita do rio Paraíba do Sul, drena uma área de aproximadamente $116 \mathrm{~km}^{2}$, estando a sua foz situada a montante da cidade de Aparecida. Ao longo dos seus $26,8 \mathrm{~km}$ de extensão, a calha principal recebe a contribuição de vários afluentes, sendo de maior expressão os córregos Branco, Mato Dentro e Roseira Velha, além de outros, de pequeno porte, que compõem a sua rede de drenagem. Os dados gerais de localização e caracterização espacial da Bacia Hidrográfica do Ribeirão Pirapitingüi, foram extraídos das cartas do IBGE com as seguintes identificações: Folhas SF-23-Y-B-IV-3 e SF23-Y-D-III-1, na escala 1:50.000.

Em termos gerais, de acordo com as condições climatológicas da região e do Estudo de Regionalização do Estado de São Paulo (DAEE, 1994), a bacia hidrográfica do Ribeirão Pirapitingüi possui precipitação anual média de $1.296 \mathrm{~mm}$ e vazão média plurianual de 1,307 $\mathrm{m}^{3} / \mathrm{s}$. (dados disponíveis na web site <http://www.sigrh.sp.gov.br>, com acesso em 08 jan. 2007).

No seu trecho inferior, caracterizado pelas várzeas do Rio Paraíba do Sul, predomina o uso agrícola, com extensas áreas de plantio de arroz. Essas áreas são divididas entre os diversos agricultores, muitos da mesma família, e nem todos possuem outorga de direito de uso, embora todos estejam cadastrados.

\section{DESCRIÇÃO DO MODELO DE SIMULAÇÃO}

O modelo desenvolvido faz a representação da vazão do curso d'água, inferindo-a se é suficiente para atender a demanda para irrigação, permitindo o ajuste dos parâmetros utilizados. Entretanto, tem-se como certo que a realidade prática dos modelos pode se deparar com diferentes condicionantes, tais como: disponibilidade de dados, aplicabilidade dos métodos, finalidades do estudo e níveis de precisão.

Com esse enfoque, o modelo foi desenvolvido com o objetivo de permitir sua utilização na bacia hidrográfica do Ribeirão Pirapitingüi; fez uso dos parâmetros minimamente necessários, mas fundamentais, para os objetivos pretendidos, tendo sido concebido com base em metodologias conhecidas, com a possibilidade de permitir seu uso em outras bacias hidrográficas de diferentes características, com a representação do comportamento do sistema dos processos envolvidos na gestão integrada dos recursos hídricos, sendo de natureza determinística (TUCCI, 1987, p. 221-222).

O modelo baseia-se num algoritmo para simular o escoamento unidimensional na bacia e a propagação das vazões de contribuição, obtido a partir das variáveis básicas da equação de um balanço hídrico que coloca em evidência as variáveis hidrológicas mais importantes, séries de vazão ou precipitação, para o intervalo de tempo e para a bacia hidrográfica, conforme os estudos de regionalização realizados pelo DAEE (1994). 
Para simular a situação do estudo de caso que orientou este trabalho, sob a forma de um processo de gestão participativa, conforme detalha, a aplicação do método permitiu reproduzir as principais características e variáveis da bacia do Ribeirão Pirapitingüi no entendimento dos múltiplos aspectos e situações envolvidos. A aplicação do método serviu, também, como instrumento de apoio para subsidiar a identificação de propostas para a tomada de decisão pelo colegiado de decisores envolvidos: órgãos de governo, comitê de bacia, responsáveis pelas outorgas de uso da água e usuários.

Com base no instrumental bibliográfico, consultas de mapas cartográficos incursões na bacia para levantar e cadastrar os pontos principais onde ocorrem as ações antrópicas, foram realizadas visitas técnicas às áreas de várzeas de cultivo de arroz irrigado, em pontos de captação e locais de conflito, e participação em encontros e eventos junto aos rizicultores e representantes do órgão gestor dos recursos hídricos responsável pela outorga e fiscalização, o DAEE.

Nesse sentido, ressalta-se a relevância dos princípios da integração e da articulação institucional das diversas políticas públicas para o êxito efetivo do modelo de gestão das águas, preconizado pela Lei $\mathrm{n}^{\circ}$ 9433/97, cabendo, aqui, resgatar o objetivo geral do Plano Nacional de Recursos Hídricos, voltado para a melhoria da oferta de água, que é gerenciar as demandas considerando ser a água um elemento estruturante para a implementação da qualidade de vida, sob a ótica do desenvolvimento sustentável e da inclusão social.

O estudo de caso da bacia hidrográfica do Ribeirão Pirapitingüi, bem como a abordagem de gestão realizados neste trabalho são emblemáticos dessa situação e confirmam, de forma dramática, uma disponibilidade que admite até 320 ha irrigados e, no confronto com ela, uma área irrigada total instalada ao longo do tempo de 1.020 ha, que justifica os conflitos ali instalados.

\section{CONCLUSÃO}

Diante do exposto, este trabalho quer contribuir para um diagnóstico objetivo da situação, com a necessária articulação setorial entre diferentes níveis de capacitação e de organização, para realizar a gestão integrada do uso múltiplo da água, já que na bacia hidrográfica do Paraíba do Sul existem muitas outras situações semelhantes a essa que ocorre na bacia hidrográfica do Ribeirão Pirapitingüi, que poderão fazer uso da metodologia implementada neste trabalho.

Assim, o processo permanente de gestão com base em monitoramento hidrológico, com o respaldo em consensos gradativos, com a adoção do modelo de ganhos compartilhados, a partir de um esquema lógico de construção para os cenários, é uma exigência imediata na bacia do Ribeirão Pirapitingüi.

Nesse contexto, pela análise do comportamento da disponibilidade versus demanda dos recursos hídricos, levando-se em conta o princípio da unicidade dos recursos, assim considerados na simulação dos cenários no intercâmbio de informações entre os rizicultores e os gestores para minimização de conflitos pelo uso da água, pode-se afirmar que a questão central da pesquisa foi alcançada e respondida.

\section{REFERÊNCIAS}

BONOMA, Tomas V. Case Research in Marketing: Opportunities, Problems, and Process. Journal of Marketing Research, Vol XXII, May, 1985.

BRAGA, B., PORTO, M., TUCCI, C. E. M. Monitoramento de Quantidade e Qualidade das Águas. $3^{\mathrm{a}}$ ed. In:_. Águas Doces no Brasil: Capital Ecológico, Uso e Conservação. São Paulo, Escrituras, 2006, p. 145-158. 
CBH-PS. Reunião Extraordinária, $18^{a}$ 2003. Cruzeiro/SP. Ata da $\mathbf{1 8}^{\mathbf{a}}$ Reunião Extraordinária do Comitê da Bacia Hidrográfica do Paraíba do Sul - CBH-PS. Secretaria Executiva do Comitê, 24/10/2003, 12 p.

CERVO, Amado Luiz. Metodologia Científica $5^{\text {a }}$ ed. Amado Luiz Cervo, Pedro Alcino Bervian. São Paulo: Prentice Hall, 2002. 241p.

FERNANDES, José Carrera; GARRIDO, Raymundo José. Economia dos recursos hídricos. 1. ed. Salvador. EDUFBA, 2002. 458p.

FREDERICK, K. D. Balancing Water Demands with Supplies; The Role of Management in a world of Increasing Scarcity. Papers 189, World Bank - Technical Papers. 1993.

GODET, M. Manual de Prospectiva Estratégica - Da Antecipação à Acção. Lisboa: Publicações Dom Quixote, 1993.

GOODE, W. J.; HATT, P. K. Métodos em Pesquisa Social. 3. ed. São Paulo: Cia Editora Nacional, 1969.

MINISTÉRIO DO MEIO AMBIENTE - Lei $\mathbf{n}^{\mathbf{0}}$ 9.433, de 8 de janeiro de 1997, in Política nacional de recursos hídricos, Secretaria Nacional de Recursos Hídricos, 2a ed. ver. atual, Brasília (DF), 1999.

MINISTÉRIO DO MEIO AMBIENTE - Plano Nacional de Recursos Hídricos Águas para o Futuro: Cenários para 2020, Volume 2/Secretaria Nacional de Recursos Hídricos/Agência Nacional de Águas, ed. Especial de lançamento, Brasília (DF), 2006.

SÃO PAULO (Estado). Portal do Governo do Estado de São Paulo. Banco de Dados. Sistema de Informações para o Gerenciamento de Recursos Hídricos do Estado de São Paulo. Regionalização Hidrológica do Estado de São Paulo. Disponível em: <http://www.sigrh.sp.gov.br>. Acesso em: 08 jan. 2007.

SÃO PAULO (Estado). Secretaria de Recursos Hídricos Saneamento e Obras. Departamento de Águas e Energia Elétrica. Plano Estadual de Recursos Hídricos. Manual de Cálculo das Vazões Máximas, Médias e Mínimas nas Bacias Hidrográficas do Estado de São Paulo. São Paulo, 1994. 63 p. (Séries Manuais).

TULL, D. S.; HAWKINS, D. I. Marketing Research, Meaning, Measurement and Method. Macmillan Publishing Co., Inc. London, 1976.

YIN, Robert K. Estudo de Caso: planejamento e métodos. / Robert K. Yin; trad. Daniel Grassi. $3^{\mathrm{a}}$ ed. Porto Alegre : Bookman, 2005. p. 212.

VALENTE, A. A. Minimização de conflitos pelo uso da água na gestão de recursos hídricos: estudo de caso na bacia hidrográfica do Ribeirão Pirapitingüi - Vale do Paraíba. Dissertação (Mestrado) - Universidade de Taubaté, Departamento de Economia, Contabilidade e Administração, 2007. 